\title{
The influence of various stimuli upon Parkinsonian tremor and rigidity ${ }^{1}$
}

\author{
JANUSZ SUBCZYNSKI, KEIZO MATSUMOTO, TUNG HUI LIN, \\ AND IRVING S. COOPER \\ From the Department of Neurosurgery, St. Barnabas Hospital, New York, New York
}

In the recent literature there have appeared several publications concerning the influence of central or peripheral stimulation upon tremor and rigidity in Parkinson's disease. Attention has mainly been directed to the effect of central electrical stimulation upon tremor. Dependent on the parameters of a given stimulus, an inhibitory or facilitatory effect was obtained (Feinstein and Levin, 1961; Jung and Hassler, 1960; Struppler, 1962). There are, however, very few clinical observations dealing with the influence of peripheral stimulation upon tremor and rigidity (Hoffman, 1962; Matsumoto, Rossman, Lin, and Cooper, 1963; Steinbrecher, 1961), and the purpose of this study was to investigate the effect of various peripheral stimuli and activities on tremor and rigidity in Parkinson's disease. The present paper deals specifically with electromyographical studies of 64 Parkinson patients.

\section{MATERIAL AND METHOD}

The age of the patients ranged from 31 to 63 with an average of 52 years. Thirty-three were men and 31 were women. All cases demonstrated bilateral symptoms which were usually more pronounced on one side.

The study was carried out in an isolated room with the patient in a sitting position with his hands placed on his knees. For the recording of tremor and rigidity an Offner type 1, 8-channel, E.E.G. machine was used. Surface electrodes were placed on the flexors and extensors of both arms. Tremor was estimated through the grouping discharges of the muscles. Rigidity was evaluated through the stretch response which may be considered as a relative index of rigidity in Parkinson's disease (Matsumoto et al., 1963).

Two kinds of stimulation were used. They were sensory (visual, acoustic, and proprioceptive) and the performance of motor and abstract motor activities (mental). For optic stimulation a stroboscope (frequency 5-6 sec.) was utilized, and for acoustic stimulation, a tunegenerator (frequency 300-600 sec.). Proprioceptive stimulation was administered by passive movements of the ipsi- and contralateral upper extremities. Motor performance

'This investigation was supported in part by a grant from the John A. Hartford Foundation, Inc. included active movements of the upper extremities Abstract motor activities consisted of counting backwards, spelling names backwards, and performing arithmetical calculations.

\section{RESULTS}

Of the patients investigated, 59 had unilateral tremor and five bilateral. In 36 cases tremor was constant, in 28 intermittent. The frequency of tremor ranged approximately from 4 to 7 per second with an average of 5.5. The frequency was fairly constant for each patient. If tremor ceased momentarily either spontaneously or by induced inhibitory manipulation and the electromyograph showed no grouping discharges when the tremor reappeared later the frequency was the same as before (Fig. 1).

Visual and acoustic stimulation had no influence upon the amplitude and frequency of tremor. On the other hand, proprioceptive stimulation by ipsilateral passive movements caused a transient inhibition of tremor immediately after stimulation (Fig. 2). This phenomenon was observed in 60 cases $(94 \%)$. The duration of inhibition seems to be in reverse proportion to the intensity of tremor and is always longer in the case of intermittent tremor.

Contralateral proprioceptive stimulation caused complete inhibition of tremor in 24 cases (37.5\%), partial in $24(37.5 \%)$, and in 16 cases $(25 \%)$ no influence was noted (Fig. 3). Here again patients with intermittent mild tremor were apt to show complete inhibition, whereas patients with constant violent tremor usually failed to demonstrate complete inhibitory effects. Inhibition manifested itself as a decrease of amplitude but not as a change of frequency of tremor.

In $87 \%$ of the group the performance of ipsilateral active movements also caused an obvious inhibitory influence upon tremor. The performance of contralateral active movements caused complete inhibition of tremor in 16 cases $(25 \%)$, partial in 11 cases $(17 \%)$, no influence in 35 cases $(55 \%)$, and exaggeration in two cases $(3 \%)$. However, in most instances 


\section{EXTENSORS \\ $-w-4-4+4-4+4-4$}
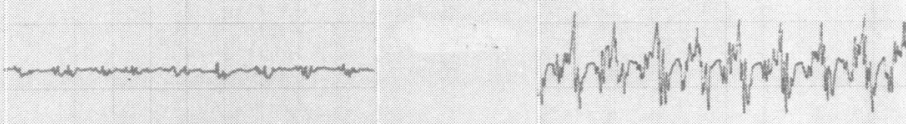

FLEXORS

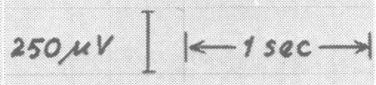

A

B

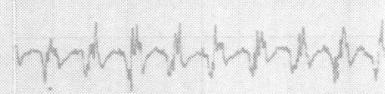

FIG. 1. E.M.G. recording of resting tremor of the same patient. A Under normal circumstances. B When relaxed. C When excited.

Notice the constant frequency of the tremor in spite of the variation of amplitude.

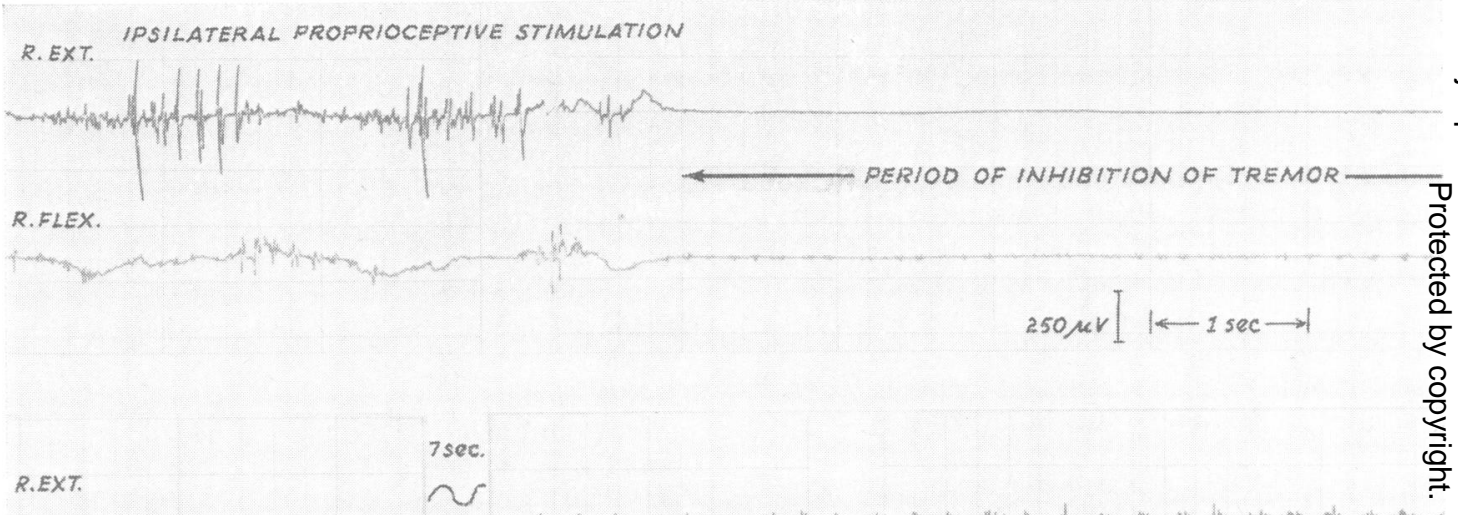

FIG. 2. Illustration of transient inhibitory phenomenon upon tremor by ipsilateral proprioceptive stimulation.

a slight decrease of amplitude of tremor for a period of 1 or 2 seconds was noted at the beginning and the end of contralateral movements (Fig. 4).

The performance of abstract motor activity (counting backwards from 100) increased the amplitude of grouping discharges in 63 cases $(98 \%)$, but, in no instance, was an increase of frequency (at least more than $1 \mathrm{sec}$.) noted (Fig. 5). In almost all cases the increase in amplitude of tremor appeared after a latency period of 2 to $8 \mathrm{sec}$. The peak of amplitude was obtained usually 15 to 30 seconds after the onset of performance. In 40 cases $(62.5 \%)$ a markedly pronounced transient decrease of amplitude of tremor was noticed after the completion of counting. In 14 cases $(25 \%)$ a slight transient decrease was observed. In 10 cases $(15.5 \%) \frac{\text { ㄱ․ }}{3}$ no immediate decrease of amplitude was found. No significant inhibitory influence of contralateralo proprioceptive stimulation was observed if performed against the background of abstract motor activities. $\stackrel{ }{-}$

Rigidity as well as stretch response was exaggerated $>$ by contralateral active movements in all investigated patients. The exaggeration of rigidity was prominent $N$ and appeared immediately after stimulation. This finding was well demonstrated in the previous study (Matsumoto et al., 1963). Contralateral propriocep- $c$ 


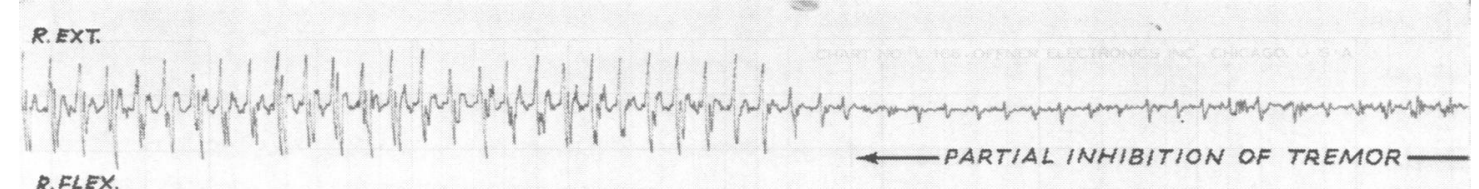

R.FLEX.

$250 \mu \mathrm{r}[\mid \leftarrow$ sec $\rightarrow$

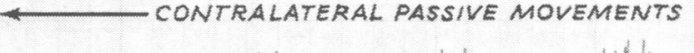

\section{1}

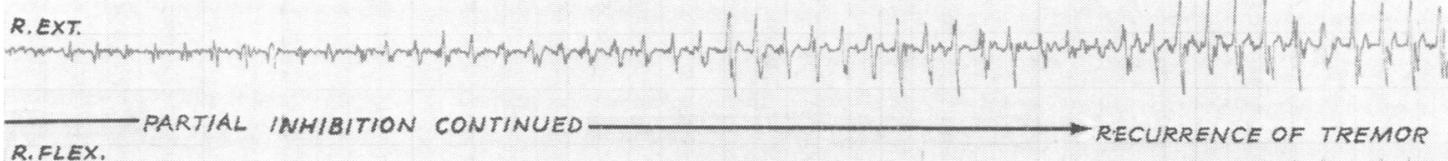

R. FLEX.
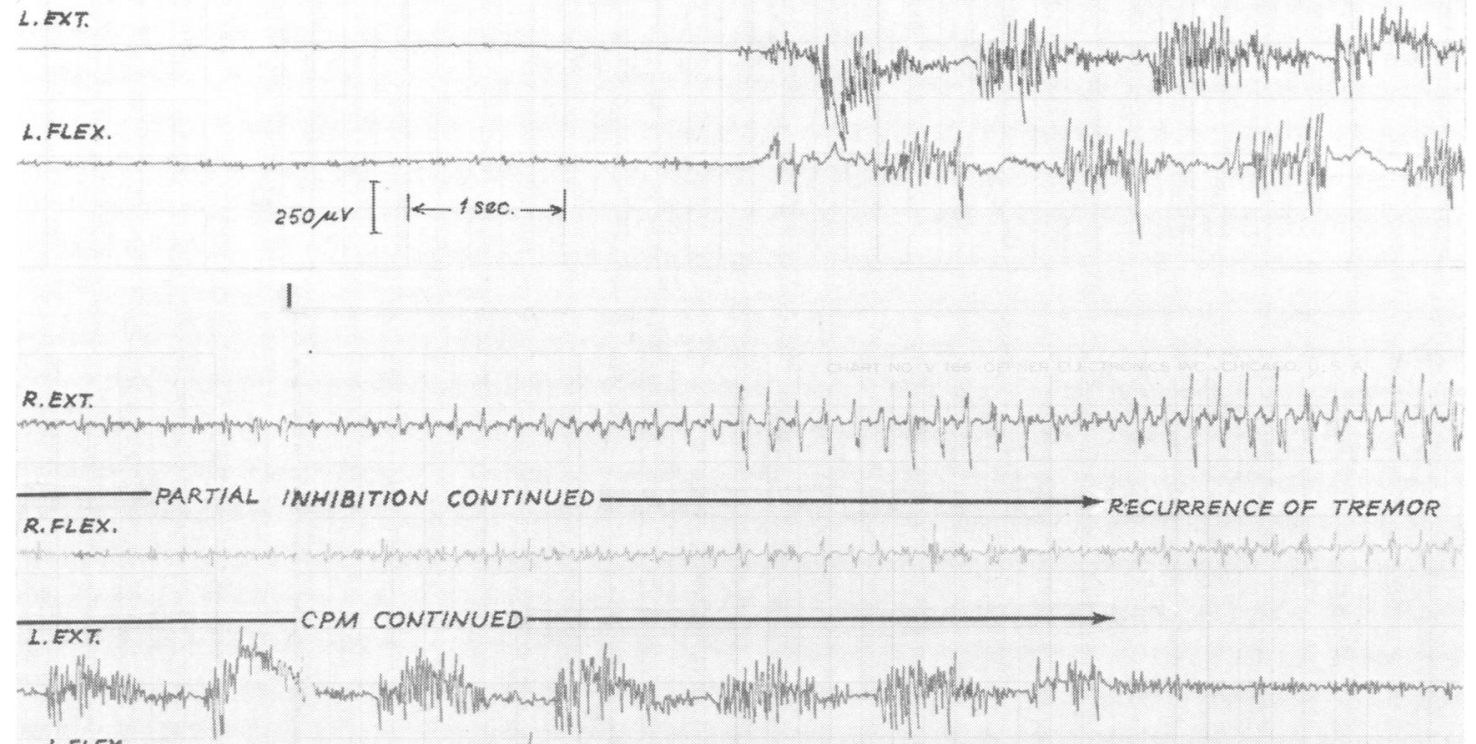

L.FLEX.

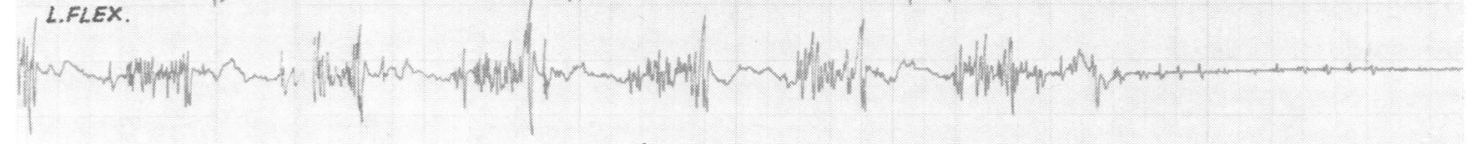

FIG. 3. Partial inhibition of tremor by contralateral movements.

TABLE I

RESULTS OF STIMULATION ON RIGIDITY AND TRFMOR

\begin{tabular}{lll} 
Kind of Stimulus & Rigidity & Tremor \\
\hline Visual & $\mathrm{O}$ & $\mathrm{O}$ \\
Acoustic & $\mathrm{O}$ & $\mathrm{O}$
\end{tabular}

Change of proprioceptive feedback

Ipsilateral

Not investigated

Contralateral

$\mathrm{O}$

Motor performance

Ipsilateral

Contralateral

Not investigated

$++$

'Mental performance'

$\mathrm{O}=$ No influence $;-=$ inhibitory influence; $+=$ exaggerated influence. tive stimulation by passive movement had no influence on stretch reflex response (Fig. 6). Visual and acoustic stimulation failed to influence rigidity in any case. The results are summarized in Table I.

\section{DISCUSSION}

It has been stated that the frequency of Parkinsonian tremor is fairly constant (Steinbrecher, 1961). This phenomenon was also observed in this study. As a matter of fact, it seems impossible to influence the frequency of tremor with the different stimuli used in this investigation.

Schreiner, MacCarty, and Grindlay (1958) described the inhibitory influence of ipsilateral active movements upon experimentally induced tremor at 


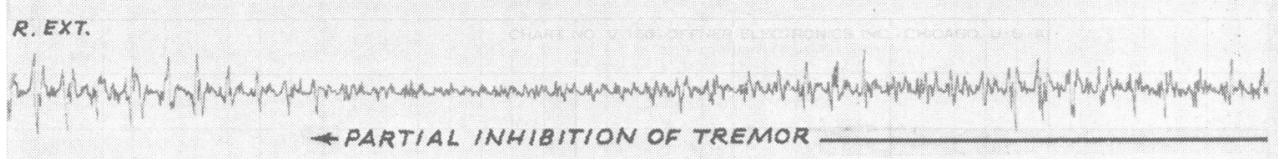

R. FLEX.

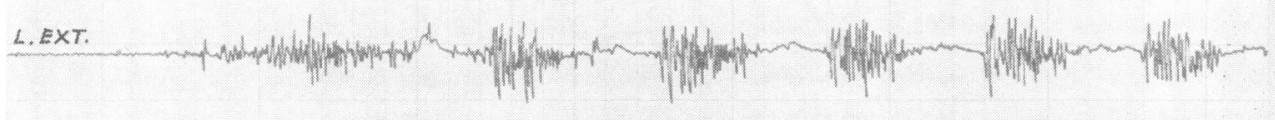

FIG. 4. Partial inhibitory influence upon tremor by contralateral active movements. Notice inhibitory phenomenon occurring at the beginning and termination of active movements.

rest in monkeys. Hoffman (1962) found that tremor in Parkinson's disease was influenced by proprioceptive input in each level of the spinal cord. Steinbrecher (1961) and Struppler, Struppler, and Schwab (1960) observed that contralateral passive movements had some inhibitory influence on tremor at rest and that this proprioceptive stimulation did not inhibit tremor against the background of 'mental activities'. Their opinion is that the last factor plays a more important role in the pathological mechanism of tremor than proprioceptive feedback. Our investigation demonstrates that proprioceptive stimulation has an inhibitory influence upon tremor at rest. On the contrary, rigidity is not influenced by such stimulation.

The inhibitory influence upon tremor is limited 을 only as to the amplitude of grouping discharges but $\frac{D}{0}$ not to the frequency. Prolonged proprioceptive stimulation produces an habituation-like phenome- N non. In time, the inhibitory effect on tremor disappears in spite of continuous stimulation and the tremor returns. Paradoxically, a sudden stopping 
R. EXT.

R, FLEX

L. EXT.

L.FLEX.

PATIENT BEGINS TO COUNT

R.EXT.

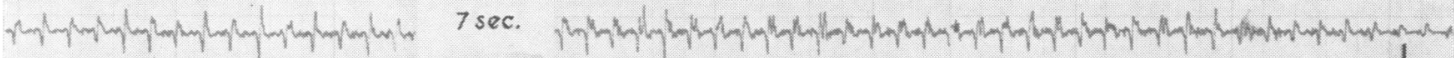
- TREAOR CONTINUED

R. FLEX.
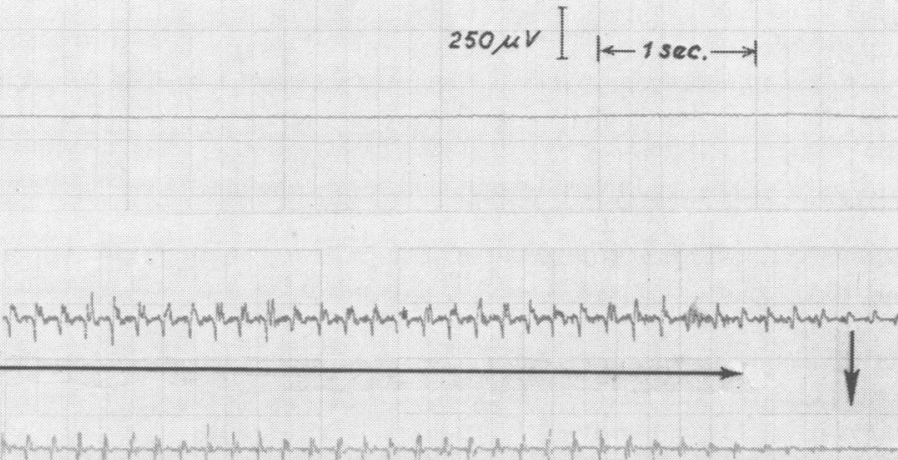

L.EXT.

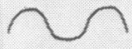

L.FLEX.

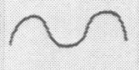

FIG. 5. Elicited tremor by performance of abstracted motor activity. Notice latent period in the beginning and transient decrease of amplitude (marked by arrow) when counting stopped.

R.EXT. $250 \mu \mathrm{V}]$ ISEC. $\longrightarrow$ CONTRALATERAL PASSIVE MOVEMENTS

\section{R.FLEX.}
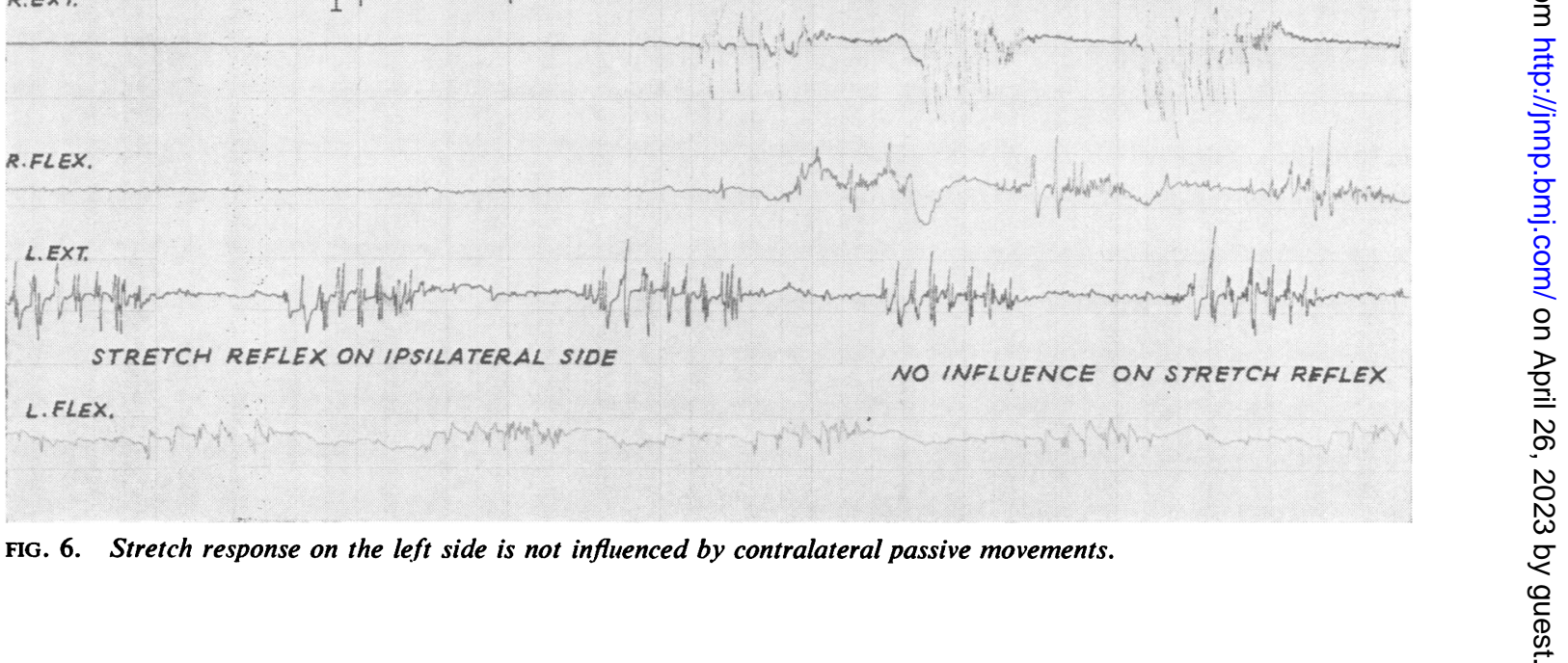
of stimulation produces an inhibitory influence upon tremor. From these observations it seems that the sudden change of proprioceptive pattern has some inhibitory influence rather than the proprioceptive stimulation itself.

The problem still remains as to the effect of mental activities upon the mechanism of tremor. The abstract motor performance seems to provoke some special 'disinhibition' or 'activation' of a pathological central mechanism responsible for the tremor at rest. Proprioceptive stimulation performed against the background of mental activities has no inhibitory effect upon tremor. Thus, the proprioceptive influences do not seem to interfere with the overwhelming fascilitatory effect of mental performance.

Simple visual and acoustic stimulations have no influence upon tremor at rest. It may therefore be postulated that stimuli without emotional meaning and not related directly to motor performance do not influence tremor.

Rigidity responds differently from tremor under the influence of various stimuli. Proprioceptive stimulation has an inhibitory influence upon tremor but does not at all modify rigidity. Mental stress produced a pronounced increase of tremor but only a slight and discrete exaggeration of rigidity. Active contralateral movements had a vague influence upon tremor but practically in all cases they produced a rapid and strong increase in rigidity. Only visual and acoustic stimulation failed to influence either tremor or rigidity.

Perhaps the most important observation made in this study is the discrepancy or the disassociation between tremor and rigidity under the influence of different stimuli. It was found that tremor could be exaggerated by mental or emotional stress and inhibited at least partially by the change of proprioceptive feedback. On the contrary, rigidity could not be influenced by any kind of proprioceptive stimuli. It seems that active motor performance has a specific facilitatory influence upon rigidity. The increase of rigidity is practically immediate, strongly pronounced, and constant in all cases. It can also be obtained by mental or emotional stress, but under these circumstances the increase is after a long latent period and to a lesser degree.
On the basis of these findings, it seems possible to conclude that in the pathological mechanism of tremor proprioceptive feedback plays an important role. On the other hand, rigidity, according to results obtained in this study, seems to be less dependent on proprioceptive feedback and is 0 primarily related to the efferent mechanism of motor performance.

SUMMARY

The influence of different stimuli upon tremor and rigidity in 64 Parkinsonian patients was electromyographically investigated. The following results were obtained:

Tremor and rigidity behaved differently under the influence of diverse peripheral stimuli. The alteration of of proprioceptive input had an inhibitory influ- $\overrightarrow{0}$ ence upon tremor at rest but not upon rigidity. Active contralateral movements exaggerated rigidity but had random and vague inhibitory influences upon tremor.

Mental stress produced a strong exaggeration of tremor and to a lesser degree of rigidity. Visual ante acoustic stimulation did not influence either rigidity iv or tremor.

On the basis of results obtained it appears thaif rigidity is less dependent upon proprioceptive feedback than is tremor. In the latter a pathologically $c$ altered proprioceptive feedback seems to play more important role.

\section{REFERENCES}

Feinstein, B., and Levin, G. (1961). Observations on subcortical stimulation in stereotactic intracerebral surgery. (Second International Congress of Neurological Surgery), Washington, 1961, Excerpta med. int. Congr. Ser., No. 36, p. E76.

Hoffman, W. W. (1962). J. Neurol. Neurosurg. Psychiat., 25, 109. (1962). Ibid., 25, 203.

Jung, R., and Hassler, R. (1960). In Handbook of Physiology, edited by J. Field, chap. 35, sect. 1. Neurophysiology, vol. 2, pp. 863927. American Physiological Society, Washington.

Matsumoto, K., Rossman, F., Lin, T. H., and Cooper, I. S. (1963). J. Neurol. Neurosurg. Psychiat., 26, 27.

Schreiner, L., MacCarty, C. S., and Grindlay, J. H. (1958). Pathogenesis and Treatment of Parkinsonism, chap. 5, edited by W. S. Fields. Thomas, Springfield, Ill.

Steinbrecher, W. (1961). Klin. Wschr., 39, 679.

Struppler, A., Struppler, E., and Schwab, R. S. (1960). Dtsch. Z. Nervenheilk., 181, 280.

-. (1962). Personal communication. 